\title{
Effets combinés de l'apport d'acide caproïque et de la concentration en protéines sur l'utilisation de laits artificiels par l'agneau préruminant 1 - Utilisation digestive des principaux constituants du régime
}

\author{
B. AUROUSSEAU, M. VERMOREL et M. THERIEZ *
}

avec la collaboration technique de F. Duborsset, J.P. Donnat, S. Gasnet, G. Moins et R. SoucheT

I.N.R.A., C.R.Z.V. de Theix

Laboratoire d'Etude du Métabolisme énergétique

* Laboratoire d'Etude de la Production ovine 63122 Ceyrat

\section{Résumé}

Les effets de la nature des lipides (suif-coprah, "SC», ou suif-coprah tricaproïne, « $\mathrm{SCT} \gg)$, de la teneur en protéine (moyenne, « $\mathrm{M} \gg$, c.à.d. 24 p. 100 de la matière sèche, "MS », ou haute, « $\mathbf{H} »$, c.à.d. 31 p. 100 de MS) ou de la quantité de tricaproïne utilisée (4,5 ou 11,4 p. 100 de MS) sur l'utilisation digestive de laits de remplacements ont été comparés au cours de 3 essais successifs mettant en jeu 56 agneaux préruminants. La digestibilité a été mesurée à 2 et 5 semaines (essai 1,16 agneaux limousins), à 1,2 et 3 semaines (essai 2, 24 agneaux limousins) et à 2 et 4 semaines (essai 3, 16 agneaux Ile-de-France).

Dans le cas des laits "SC », la digestibilité de MS, E (énergie) et N (azote) augmente de façon linéaire $(P<0,005)$ entre la première semaine (CUDa de 95,5, 94,6 et 95,1 p. 100 respectivement) et la troisième semaine (CUDa de 98,0, 97,6 et 97,0 p. 100 respectivement). Pour ce type de laits, la digestibilité des 3 constituants s'accroît avec la teneur en protéines $(\mathrm{P}<0,05)$ chez les animaux les plus jeunes (1 et 2 semaines) de 95,3, 94,2 et 95,0 p. 100 à $96,8,97,1$ et 96,3 p. 100 respectivement pour les laits « $\mathrm{SCM}$ » ou « $\mathrm{SCH}$ ».

Dans le cas des laits «SCT », la digestibilité des 3 constituants n'est pas influencée par l'âge des animaux ou la teneur en protéines des laits; en comparaison des valeurs observées dans le cas des laits « $\mathrm{SC}$ », elle est accrue $(\mathrm{P}<0,05)$ chez les animaux les plus jeunes ( 1 et 2 semaines) de $96,1,95,6$ et 95,5 p. 100 à $98,4,98,3$ et 97,0 p. 100 respectivement pour MS, E ou N.

Dans le cas des laits "SCM» et chez les animaux de 1 semaine, la digestibilité des 3 constituants est corrélée positivement avec le poids de naissance des agneaux $(r=0,70)$ et inversement avec leur vitesse de croissance dans le cas de MS et de E $(\mathrm{r}=-0,86)$. A tous les autres âges ou pour tous les autres régimes, aucune relation ne relie la digestibilité de MS, $\mathrm{E}$ ou $\mathbf{N}$ au poids de naissance ou à la vitesse de croissance des animaux.

\section{Introduction}

De nombreux travaux consacrés aux mécanismes de la digestion et de l'absorption des triglycérides (TG) d'acides gras à chaîne moyenne (AGCM) ont montré l'intérêt de ces composés pour l'alimentation du jeune animal. Ils présentent la parti- 


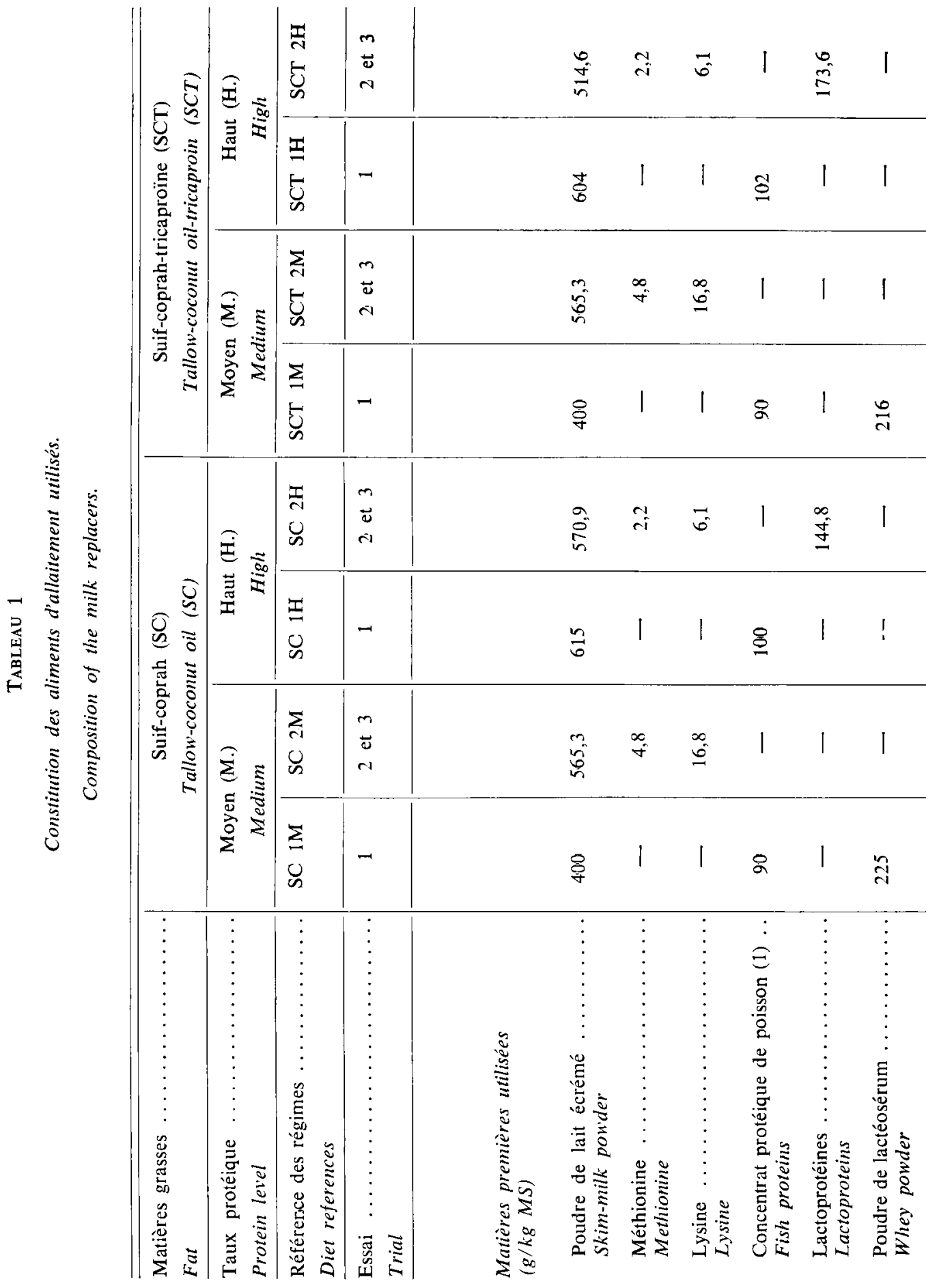




\begin{tabular}{|c|c|c|c|c|c|c|c|c|c|}
\hline $1 \quad \frac{n}{\infty}$ & $\stackrel{\forall}{\mathscr{\infty}}$ & $\stackrel{0}{E}$ & $\begin{array}{l}0 \\
0 \\
0 \\
\pm\end{array}$ & $\hat{\theta}$ & $\underline{I}$ & $\begin{array}{l}\text { J } \\
\text { i }\end{array}$ & $\begin{array}{c}2 \\
\infty \\
y\end{array}$ & م) & $\stackrel{n}{=}$ \\
\hline
\end{tabular}

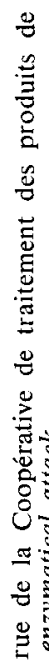

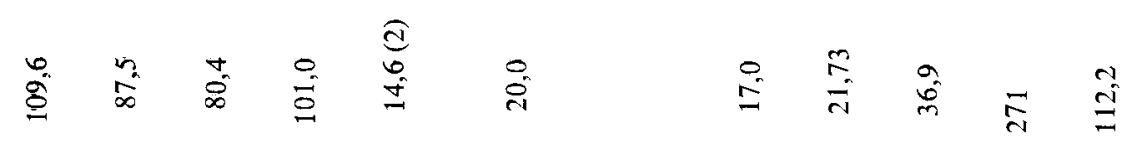

$12 \approx 2 \stackrel{0}{=}$

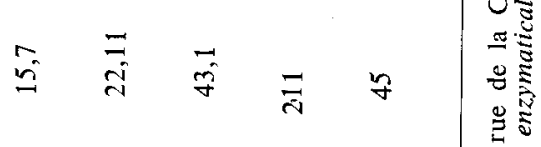

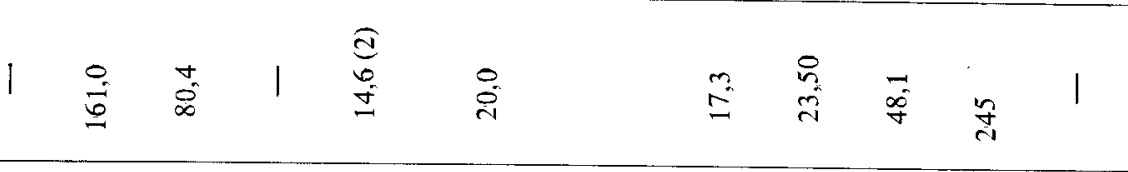

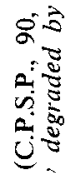

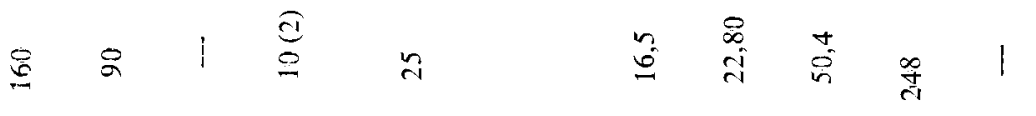

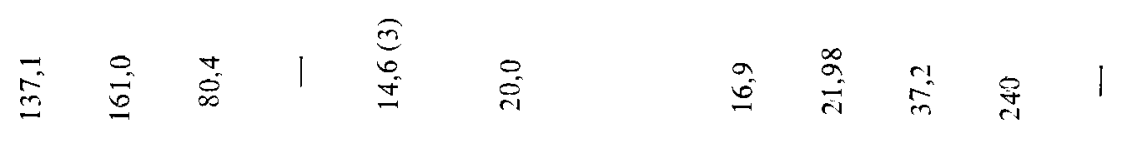

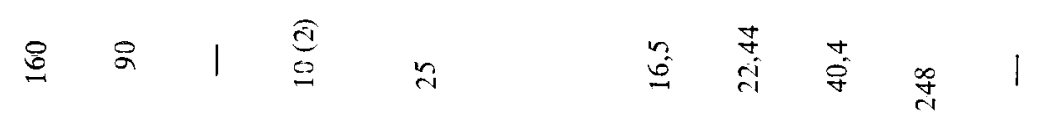

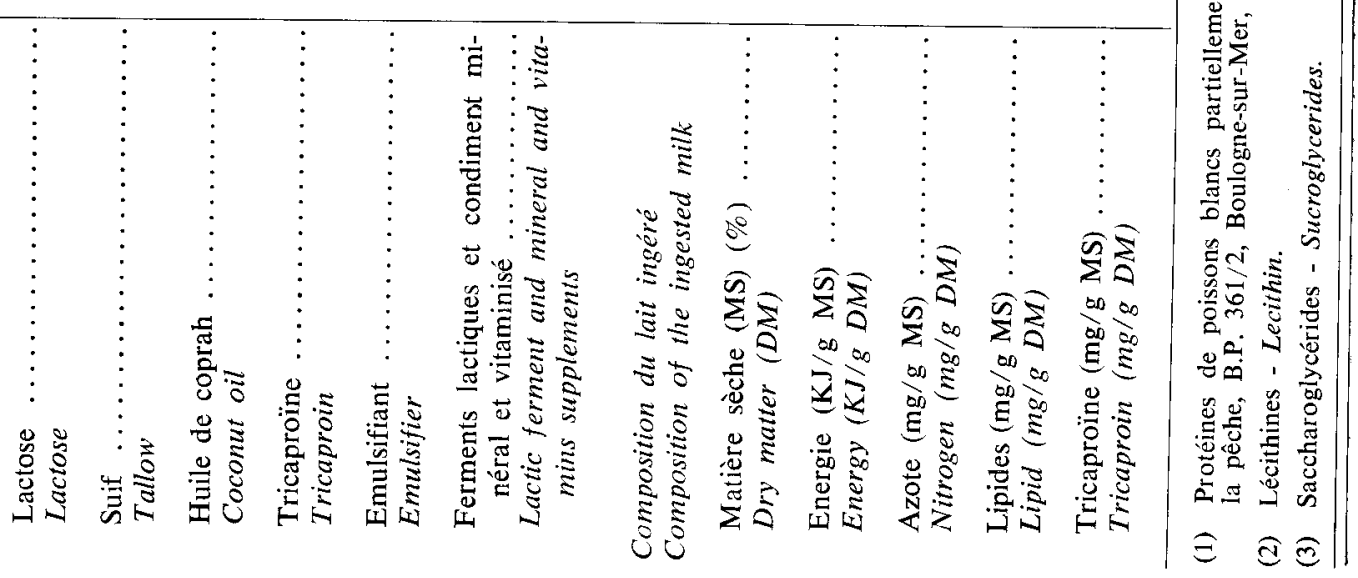


cularité d'être efficacement hydrolysés par la lipase salivaire dans la caillette du jeune préruminant, agneau (Leat \& Harrison, 1975) ou veau (EdWards-WebB \& Thompson, 1977). Une proportion importante des acides gras les plus courts (acide butyrique, acide caproïque) peut être absorbée aussitôt à travers les parois de la caillette (EDWARDSWeвв \& ThOMPSON, 1978). Les triglycérides d'AGCM sont également plus rapidement hydrolysés sous l'action de la lipase pancréatique que les TG d'acides gras longs (ENTRESSANGles et al., 1961). Enfin, la présence de tricaproïne ou d'huile de coprah dans les aliments d'allaitement offerts au très jeune veau préruminant améliore la digestibilité des régimes et accroît la fixation de protéines dans les tissus (Aurousseau, VERMOREL \& BOUVIER, 1983).

Il paraissait intéressant d'approfondir ces observations et d'étudier les variations des effets nutritionnels de l'ingestion de tricaproïne selon l'âge des animaux ou la concentration en matières azotées des aliments d'allaitement. L'agneau préruminant présente le double intérêt d'une grande vitesse de maturation physiologique et d'un petit format; il a donc éét choisi comme animal expérimental. Nous rapportons ici les résultats concernant l'utilisation digestive des aliments.

\section{Matériel et méthodes}

\section{A. Aliments}

L'utilisation digestive de 8 aliments d'allaitement a été étudiée au cours de 3 essais successifs. Les aliments utilisés pour cette étude (tabl. 1) se différencient par 3 facteurs :

- la nature des matières grasses : mélange suif-coprah (SC) ou mélange suifcoprah-tricaproïne (SCT) ;

- la concentration azotée : moyenne, M (environ 24 p. 100 de la matière sèche, MS) ou élevée, $H$ (environ 31 p. 100 de MS);

- Ia teneur en tricaproïne : 4,5 p. 100 de MS dans l'essai 1 ou 11,4 p. 100 de MS dans les essais 2 et 3.

Dans l'essai 1, les 4 aliments d'allaitement comportaient de la poudre de lait écrémé et des quantités voisines de concentrat protéique de poisson. Les aliments à teneur élevée en matières azotées (SC $1 \mathrm{H}$ ou SCT $1 \mathrm{H}$ ) étaient obtenus en remplaçant la poudre de lactosérum des aliments à teneur moyenne (SC $1 \mathrm{M}$ ou SCT $1 \mathrm{M}$ ) par de la poudre de lait écrémé.

Dans les essais 2 et 3, les aliments ne comportaient pas d'hydrolysat de poisson. Pour obtenir les aliments à teneur élevée en matières azotées (SC $2 \mathrm{H}$ ou SCT 2H), le lactose des aliments à teneur moyenne (SC $2 \mathrm{M}$ ou SCT $2 \mathrm{M}$ ) a été remplacé par des lactoprotéines obtenues par ultrafiltration. Les 4 aliments d'allaitement étaient supplémentés en méthionine et en lysine de façon à obtenir des apports identiques dans tous les cas et à assurer la satisfaction des besoins tels qu'ils ont été définis par Patureau-Mirand et Theriez (1977). 
Pour des raisons matérielles, dans l'essai 1, la tricaproïne était ajoutée aux aliments d'allaitement au moment de la reconstitution du lait artificiel. Une partie de la tricaproïne a surnagé et le lait ingéré a été ainsi indirectement enrichi en protéines (tabl. 1). En revanche, dans les essais 2 et 3, la tricaproïne était intérestérifiée à du suif avant d'être incorporée dans l'aliment d'allaitement séché ensuite sur tour Spray. Le mélange de matières grasses distribué et ingéré par les agneaux comportait ainsi, sur la base de l'énergie brute, $1 / 3$ de suif, $1 / 3$ de coprah et $1 / 3$ de tricaproïne. La différence de concentration en énergie de la tricaproïne, comparativement aux TG d'acides gras longs, a été prise en compte et les apports de matières azotées dans les aliments rééquilibrés en conséquence.

\section{B. Animaux}

L'ensemble du schéma expérimental et le détail des périodes de mesure de la digestibilité des aliments au cours des 3 essais successifs sont représentés sur la figure 1.

Race des agneaux Breed of the lambs

Mesures

Meosurements

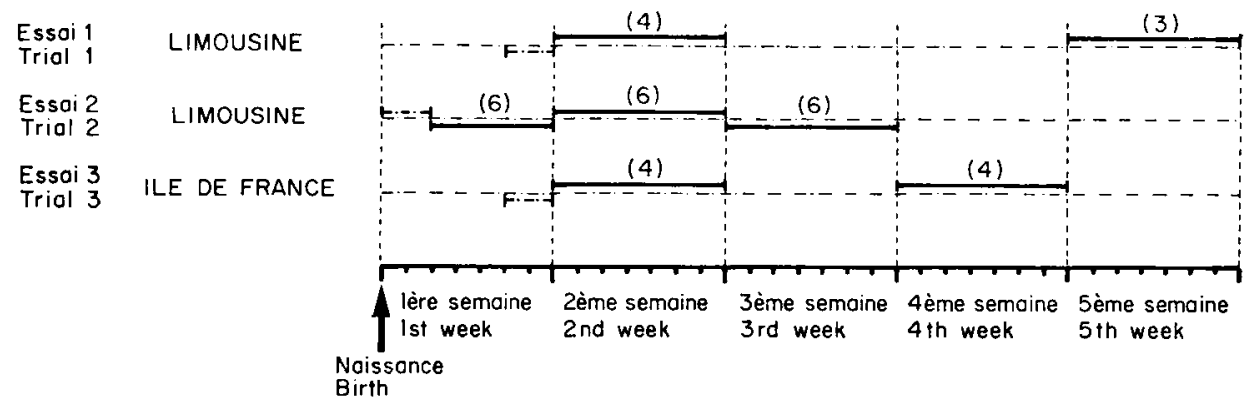

FIG. 1

Schéma expérimental.

Experimental scheme

( $₹:=:-4$ période d'adaptation ; F.- $\rightarrow$ période de collecte des fèces et des urines). adaptation period; faeces and urine collection periods.

(n) Nombre dagneaux par traitement - Number of lambs in each group.

Dans un premier essai, 16 agneaux mâles de race limousine ont été séparés de leur mère après la première tétée de colostrum. Ils ont été adaptés à la consommation de lait artificiel à la tétine, au sol, en groupe. Ils ont ensuite été installés dans des cases individuelles, attachés, adaptés au port de harnais destinés à la récolte des fèces (Tissier, Bechet \& Molenat, 1975) et répartis en quatre groupes homologues à l'âge de 5 à 6 jours. Ils ont été utilisés pour la détermination des caractéristiques d'utilisation digestive et métabolique des régimes aux âges de 2 semaines (4 animaux par régime) et 5 semaines ( 3 animaux par régime). 
Dans un second essai, 24 agneaux mâles de race limousine ont été utilisés au cours de deux répétitions. Les agneaux séparés de leur mère après la première tétée ont été immédiatement adaptés au port des harnais et à la contention en cage individuelle. La digestibilité et la rétention azotée des aliments ont été déterminées avec ces agneaux répartis en 4 groupes homologues depuis la naissance jusqu'à un âge moyen de 22 jours ( 3 périodes de 7 jours).

Parallèlement aux mesures sur agneaux limousins, on a étudié dans un troisième essai l'utilisation des mêmes aliments par des agneaux de race Ile-de-France (4 animaux par groupe) au cours de la deuxième et de la quatrième semaine de vie.

\section{Mesures et analyses}

Les laits artificiels ont été offerts ad libitum à raison de 2 distributions quotidiennes dans des flacons suspendus à l'extérieur des cages et laissés à la disposition des agneaux jusqu'à la distribution suivante. Les agneaux consommaient leurs rations à l'aide de tétines fixées à l'intérieur des cages et prolongées par des tuyaux souples plongeant dans les flacons.

Les quantités d'aliment distribuées et refusées ont été contrôlées de façon systématique. Des échantillons représentatifs de l'aliment distribué ou des refus étaient constitués pour chaque période à partir des prélèvements effectués deux fois par jour au moment des distributions. Une partie aliquote de ces échantillons était séchée en deux temps, par lyophilisation puis passage à l'étuve $\left(24 \mathrm{~h}\right.$ à $\left.100^{\circ} \mathrm{C}\right)$ avant d'être homogénéisée dans un mortier.

Les fèces ont été collectées à l'aide de béchers assujettis aux harnais directement collés sur la toison des animaux. Elles ont été récoltées chaque jour pendant 5 à 7 jours, pesées et stockées à l'abri de l'air à la température de $-15^{\circ} \mathrm{C}$. Elles ont été ensuite séchées à l'étuve ( $48 \mathrm{~h}$ à 100 "C) avant d'être réduites en poudre à l'aide d'un broyeur à hélice de type Vertec.

Les teneurs en matières sèches des différents échantillons ont été déterminées par contrôle des pertes de poids lors de leur séchage et leurs teneurs en énergie et en azote respectivement à l'aide d'un calorimètre adiabatique Gallenkamp ou selon la méthode Kjeldahl.

Les données ont été soumises à une analyse de variance, selon une classification à 3 voies (effets de l'âge des agneaux, de la teneur en MAT ou de la nature des matières grasses du lait) avec des effectifs différents (SNEdECOR \& Cochran, 1971).

\section{Résultats}

\section{A. Variation de la digestibilité des aliments avec l'âge des agneaux}

L'âge des agneaux est un facteur important de l'efficacité de l'utilisation digestive des laits qu'ils consomment $(\mathrm{P}<0,005)$ et explique respectivement 17,17 ou 16 p. 100 de la variabilité totale de la digestibilité de la matière sèche, de l'énergie ou de l'azote (tabl. 2). Les interactions de l'âge des animaux avec la nature des lipides utilisés jouent 


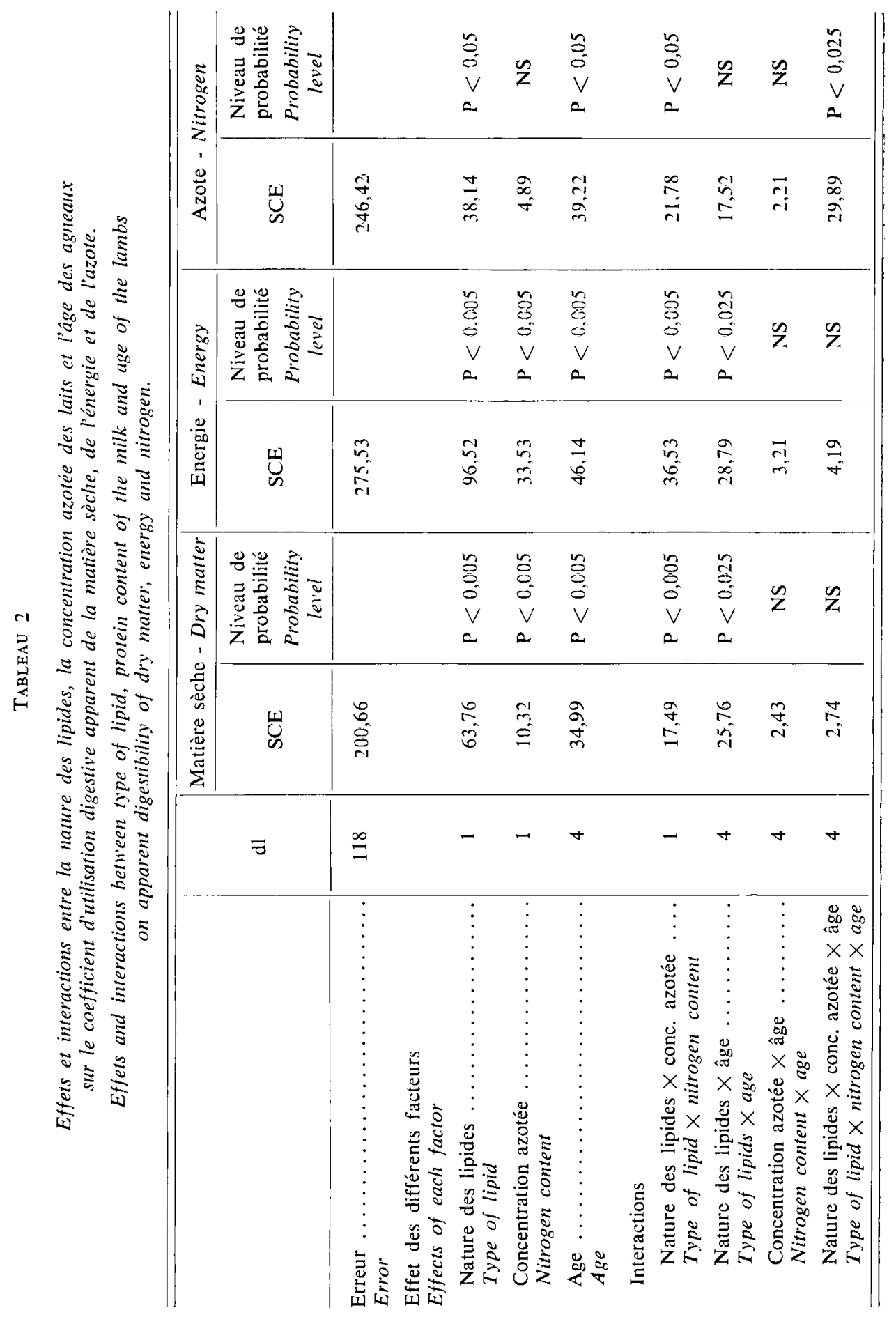




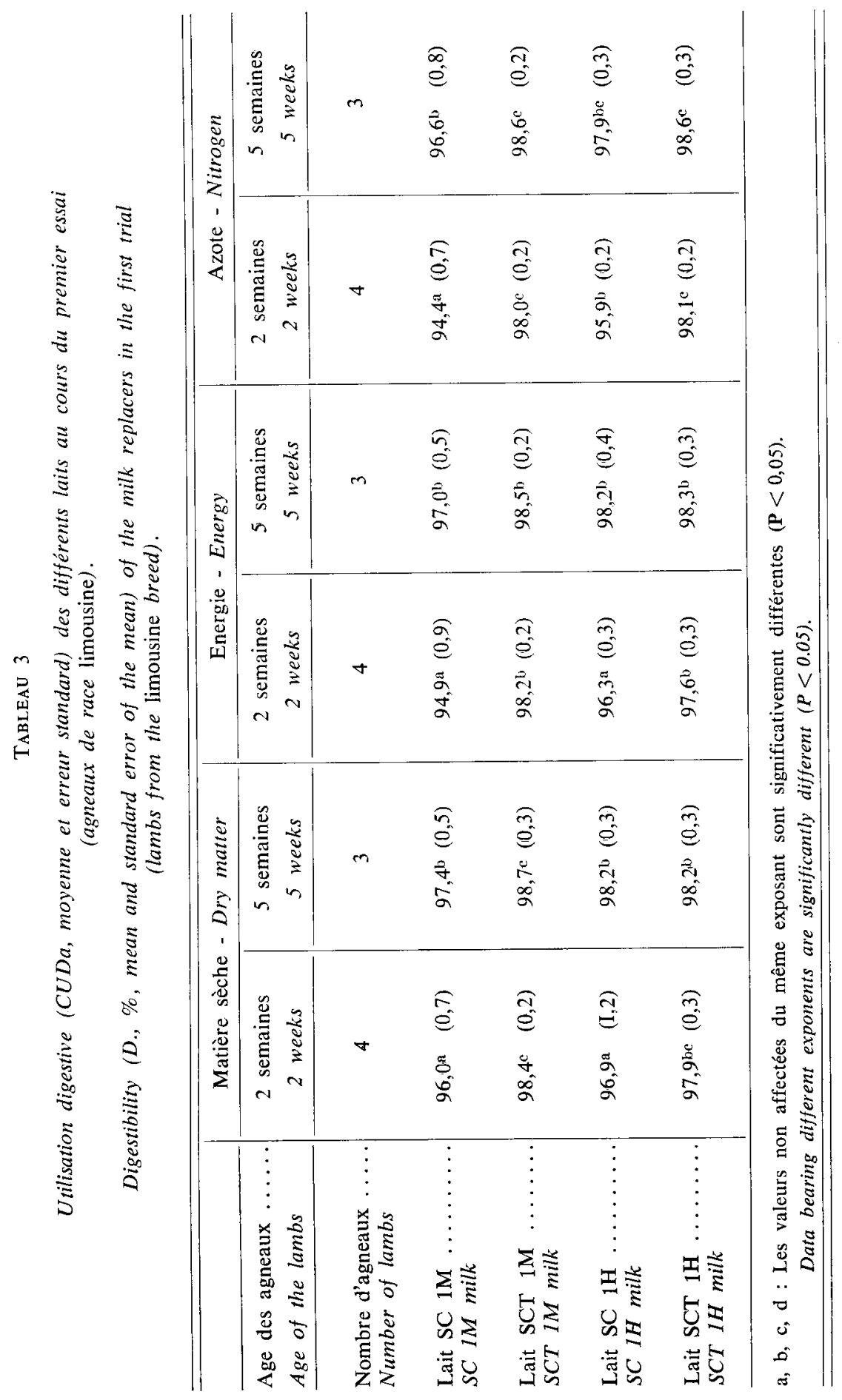


également un rôle important dans le cas de la variabilité de la digestibilité de la matière sèche et de l'énergie $(P<0,025)$ dont elles expliquent 13 ou 10 p. 100 supplémentaires respectivement. Par ailleurs, les interactions combinées de l'âge des animaux, de la concentration azotée des laits et de la nature des matières grasses utilisées expliquent 12 p. 100 de la variabilité totale de la digestibilité des matières azotées $(\mathbf{P}<0,025)$. Ainsi, dans le cas des agneaux limousins, l'utilisation digestive des aliments d'allaitement «suif-coprah » a augmenté de façon systématique avec l'âge. Dans l'essai 1, le coefficient d'utilisation digestive apparent (CUDa) a été amélioré significativement en moyenne de 1,3 point $(\mathrm{P}<0,01)$ pour la matière sèche (MS) de 2,0 points $(\mathrm{P}<0,005)$ pour l'énergie $(\mathrm{E})$ et de 2,1 points $(\mathrm{P}<0,001)$ pour l'azote $(\mathrm{N})$ entre la deuxième et la cinquième semaine (tabl. 3) ; l'augmentation a été identique pour les deux niveaux de concentration azotée mis en œuvre (aliments SC $1 \mathrm{M}$ et SC $1 \mathrm{H}$ ). Dans l'essai 2, le phénomène est comparable (fig. 2). Les CUDa de MS, E ou N augmentent à peu près linéairement de la première à la troisième semaine (de $2,5,3,0$ et 1,9 points respectivement $(\mathrm{P}<0,005)$ en moyenne pour les deux types d'aliments (SC $2 \mathrm{M}$ et $\mathrm{SC} 2 \mathrm{H}$ ).

Dans le cas des agneaux Ile-de-France, l'utilisation digestive de l'aliment SC $2 \mathrm{M}$ (tabl. 4) est voisine de celle observée chez les animaux limousins. Cependant, les augmentations des CUDa, entre la deuxième et la quatrième semaine $(1,6,1,1$ et 1,7 point respectivement pour $\mathrm{MS}, \mathrm{E}$ ou $\mathrm{N}$ ) ne sont pas significatives. De plus, l'utilisation digestive de l'aliment SC $2 \mathrm{H}$ n'évolue pratiquement pas, chez les animaux de ce génotype, de la deuxième à la quatrième semaine.

Enfin, la digestibilité des laits «suif-coprah-tricaproine» n'est pas significativement influencée par l'âge des agneaux.

\section{B. Variation de la digestibilité des aliments avec leur teneur en matières azotées}

La teneur en matières azotées des aliments est également un facteur important de la digestibilité de la matière sèche et de l'énergie dont elle explique à elle seule 5 ou 10 p. 100 respectivement de la variabilité totale $(P<0,005)$ mais 9 et 13 p. 100 supplémentaires, en interaction avec la nature des matières grasses utilisées $(P<0,005)$. Ses effets sur la digestibilité de $N$ (tabl. 2) sont masqués par les interactions avec la nature des lipides (explication de 9 p. 100 de la variabilité totale, $P<0,005$ ) ou avec à la fois la nature des lipides et l'âge des animaux (12 p. 100 supplémentaires, $\mathbf{P}<0,025)$.

Chez les animaux limousins, dans l'essai 1, la digestibilité des différents constituants alimentaires est supérieure de 0,8 à 1,5 point pour l'aliment $\mathrm{SC} 1 \mathrm{H}$ comparé à l'aliment SC 1M. Cependant, l'amélioration du CUDa n'atteint le seuil de signification $(P<0,05)$ qu'en $2^{2}$ semaine et dans le cas de l'azote (tabl. 3). Dans l'essai 2, l'augmentation de la concentration en matières azotées des laits de 24 p. 100 (aliment SC 2M) à 30 p. 100 (aliment $\mathrm{SC} 2 \mathrm{H}$ ) provoque une amélioration significative $(\mathrm{P}<0,05)$ du CUDa de MS en première semaine (+ 2,3 points). Le CUDa de l'énergie est amélioré en moyenne de 2,8 points $(P<0,05)$ au cours de chacun des deux premières semaines. Celui de l'azote, enfin, est augmenté de 0,7 point seulement en première semaine (NS), et de 1,7 point $(P<0,05)$ en deuxième semaine. En troisième semaine, les différences d'efficacité d'utilisation digestive des deux types d'aliments $(0,7,1,2$ et 0,9 point respectivement pour $\mathrm{MS}, \mathrm{E}$ ou $\mathrm{N}$ ) ne sont plus significatives. 


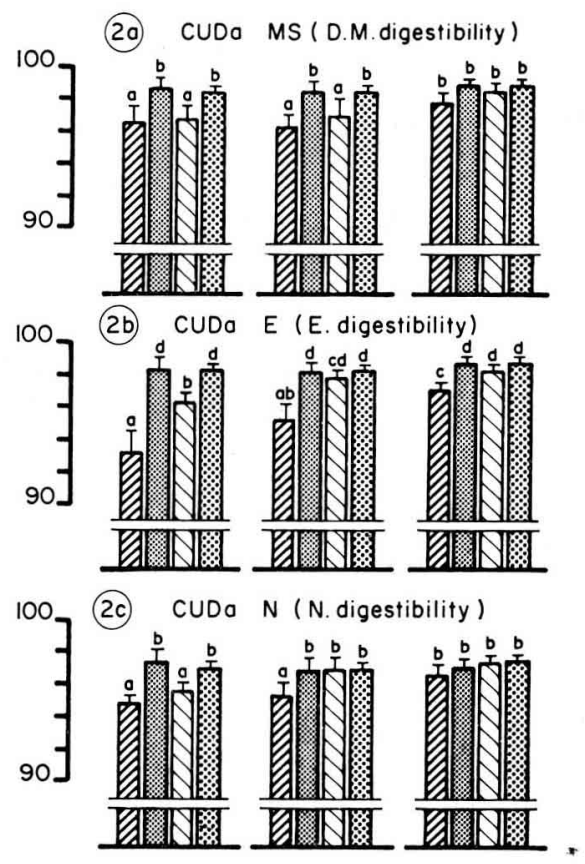

FIG. 2

Influence combinée de l'ingestion d'acide caproique ou de la teneur du lait en protéines sur la digestibilité de la matière sèche, de l'énergie et de l'azote (moyenne et erreur standard) chez l'agneau préruminant âgé de I, 2 ou 3 semaines

Combined effects of caproic acid intake and protein content of the milk on digestibility of dry matter, energy and nitrogen (mean and standard error of the mean) in the preruminant lamb of 1,2 or 3 weeks of age.

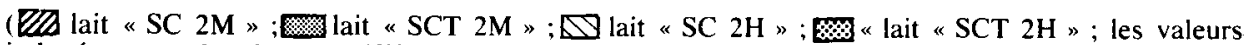
indexées par des lettres différentes sont significativement différentes: ab, bc, cd, $p<0,05$; bd, $\mathrm{p}<0,01 ;$ ad, $\mathrm{p}<0,001)$.

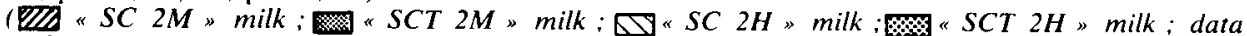
with different letters are significantly different $=a b, \quad b c, c d, p<0,05 ; \quad b d, \quad p<0,01$; $a d, p<0,001$ ).

Chez les agneaux Ile-de-France, enfin (essai 3), la digestibilité de l'aliment SC $2 \mathrm{H}$ est également supérieure, en deuxième semaine $(P<0,05)$ à celles observées dans le cas de l'aliment SC $2 \mathrm{M}$. Les CUDa respectifs de MS, E et $\mathrm{N}$ sont améliorés de 2,3, 2,7 et 2,9 points. En quatrième semaine, seul le CUDa de l'énergie est encore significativement amélioré $(+1,5$ point, $\mathrm{P}<0,05)$ par l'accroissement de la concentration en matières azotées de l'aliment.

Par ailleurs, la digestibilité des laits comportant de lacide caproïque (aliment «SCT») n'est pas modifiée par leur teneur en matières azotées. 


\section{Variation de la digestibilité des aliments avec la nature des matières grasses utilisées}

L'analyse de variance montre (tabl. 2) que la nature des lipides est le principal facteur influençant la digestibilité des laits. Ce paramètre explique en effet à lui seul respectivement 31,35 ou 15 p. 100 de la variabilité de la digestibilité de la matière sèche, de l'énergie ou de l'azote, et intervient également en interaction avec la concentration azotée des laits, avec l'âge des agneaux et avec les deux paramètres à la fois dans le cas de la digestibilité de l'azote.

L'effet de la présence d'acide caproïque dans les aliments se caractérise principalement par une amélioration très nette de la digestibilité des aliments d'allaitement chez les agneaux les plus jeunes. De plus, l'utilisation digestive des aliments comportant de l'acide caproïque est du même ordre à tous les stades étudiés pour les deux niveaux de teneur en matières azotées des aliments.

Ainsi, chez les animaux limousins, au cours de la première semaine (essai 2), les améliorations des CUDa atteignent dans le cas des aliments à 24 p. 100 de matières azotées (lait SCT 2M comparé au lait SC 2M) un niveau de 4,2 (P<0,025), 5,0 $(\mathrm{P}<0,005)$ et 2,6 points $(\mathrm{P}<0,025)$ respectivement pour $\mathrm{MS}, \mathrm{E}$ et $\mathrm{N}$. Dans le cas des aliments à 30 p. 100 de matières azotées (lait SCT $2 \mathrm{H}$ comparé au lait SC $2 \mathrm{H}$ ) les améliorations ne sont que de 1,6, 2,0 et de 1,5 points $(\mathrm{P}<0,05)$.

Chez les animaux de ce type, les différences restent significatives $(P<0,05)$ mais se réduisent sensiblement en deuxième semaine. Les CUDa des aliments SCT $1 \mathrm{M}$ ou SCT $2 \mathrm{M}$ comparés à ceux des aliments SC $1 \mathrm{M}$ ou $\mathrm{SC} 2 \mathrm{M}$ sont ainsi améliorés en moyenne de 2,2, 3,1 et 2,5 points respectivement pour MS, $E$ et $\mathrm{N}$. Les améliorations sont plus faibles dans le cas des CUDa des aliments SCT $1 \mathrm{H}$ ou SCT $2 \mathrm{H}$ comparés à ceux des aliments $\mathrm{SC} 1 \mathrm{H}$ ou SC $2 \mathrm{H}$, et s'élèvent en moyenne à 1,2 point, 0,9 point ou 1,1 point respectivement pour les mêmes composés que précédemment.

A partir de la troisième semaine, enfin, les différences de digestibilité entre les aliments d'allaitement comportant de l'acide caproïque et ceux n'en comportant pas deviennent tout à fait négligeables chez les agneaux limousins pour les deux niveaux de concentration azotée utilisés.

Chez les animaux Ile-de-France (tabl. 4), les effets de la présence d'acide caproïque dans les aliments sont, globalement, beaucoup moins marqués que chez les agneaux limousins, mais ils gardent une amplitude voisine à 2 semaines ou à 4 semaines d'âge, dans le cas des aliments à 24 p. 100 de matières azotées (lait SCT $2 \mathrm{M}$ comparé au lait SC 2M). Les CUDa respectifs de MS, E ou $\mathrm{N}$ sont ainsi améliorés en moyenne aux deux âges de 1,7,2,2 ou 1,2 point. Les améliorations du CUDa de l'énergie sont significatives $(\mathrm{P}<0,05)$ aux deux âges. Chez les agneaux Ile-de-France, la digestibilité des aliments à 30 p. 100 de matières azotées (lait SCT $2 \mathrm{H}$ comparé au lait SC $2 \mathrm{H}$ ) n'est pas modifiée par la présence d'acide caproïque. 


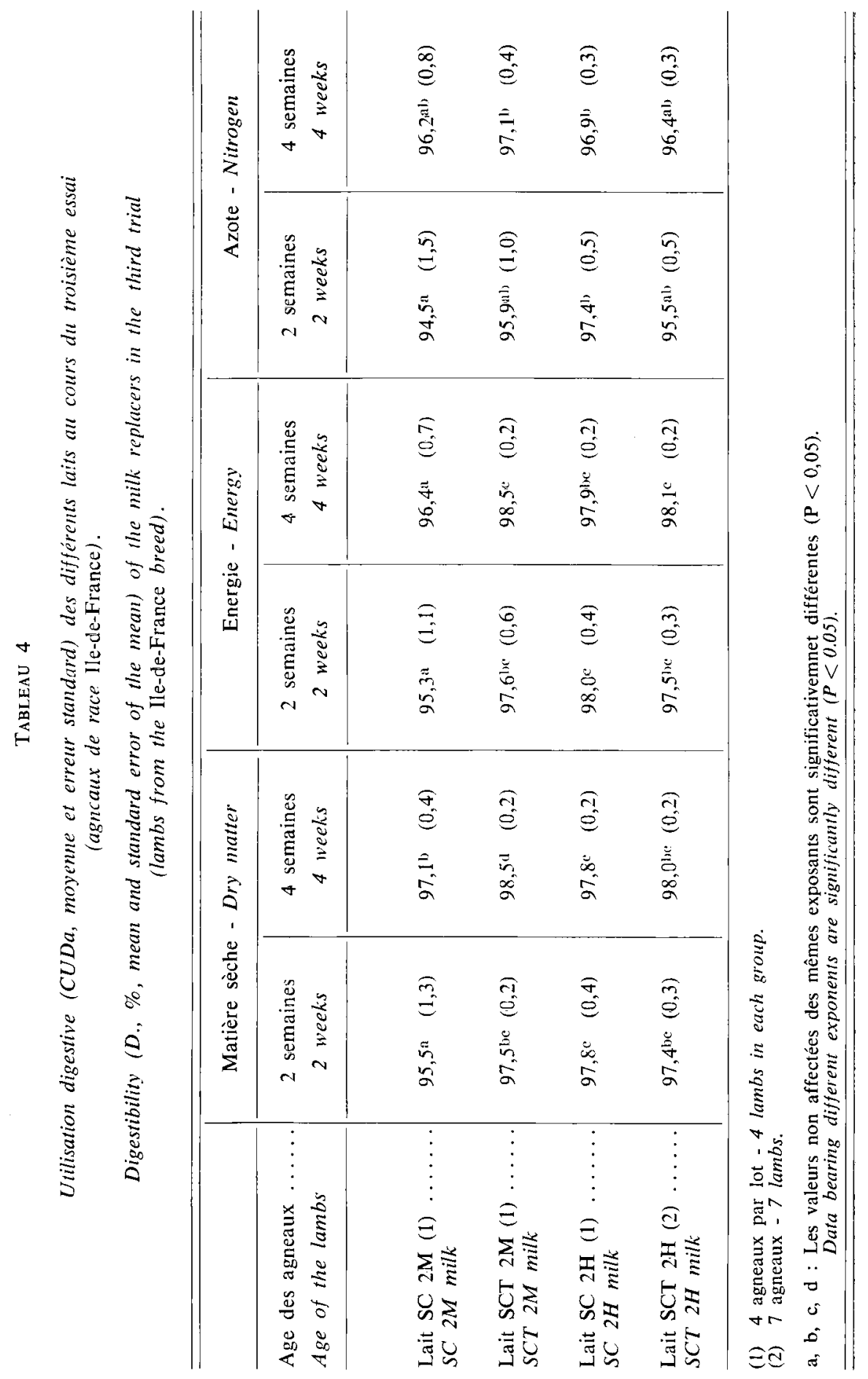




\section{Discussion}

L'amélioration de la digestibilité des aliments d'allaitement SC $1 \mathrm{M}$ et SC $2 \mathrm{M}$ observée jusqu'à l'âge de 3 semaines chez les agneaux limousins est contraire aux observations de Walker \& Cook (1967), Theriez, Protais \& Molenat (1974) mais en accord avec celles de Norton \& Walker (1971) et de Theriez, PatureauMirand \& Molenat (1977) chez l'agneau ou de Paruelle et al. (1972) et de Toullec, Frantzen \& Mathieu (1974) chez le veau. Cette amélioration des CUDa des différents principes alimentaires pourrait s'expliquer par l'évolution des sécrétions digestives. Ainsi l'action de la pepsine au niveau de la caillette du jeune veau peut être facilitée au cours des 3 premières semaines de vie de cet animal par l'accroissement de la sécrétion d'acide chlorhydrique observé pendant ce même laps de temps (TERnouTH \& Roy, 1973). L'activité protéolytique totale du suc pancréatique et les quantités de trypsine ou de chymotrypsine, sécrétées ou présentes dans le pancréas augmentent considérablement entre le deuxième jour et la troisième semaine de vie des animaux préruminants (Ternouth, Siddons \& Toothill, 1971 ; Guilloteau et al., 1983 a, 1983 b). Les quantités de lipase et de colipase présentes dans le pancréas évoluent de façon comparable à celles d'enzymes protéolytiques (Gullloteau et al., $1983 \mathrm{a}$, $1983 \mathrm{~b}$ ).

Conformément aux observations de Houssin \& Davicco (1979), l'utilisation digestive des laits SC $1 \mathrm{M}$ et SC $2 \mathrm{M}$ (comportant 24 p. 100 de matières azotées environ) est corrélée au poids de naissance, chez les agneaux de moins d'une semaine (tabl. 5). Cependant, les paramètres des régressions restent assez mal définis en raison du petit nombre d'animaux employés et de la faible variabilité de leur poids de naissance.

Les résultats rapportés ci-dessus montrent aussi que dans le cas des aliments d'allaitement de type conventionnel (teneur moyenne en matières azotées, proportions importantes de suif, les digestibilités de MS et de E (tabl. 5) sont étroitement corrélées, mais de façon négative, avec la vitesse de croissance du jeune agneau entre la naissance et l'âge de 7 à 8 jours. Il n'en est pas de même pour les matières azotées. Les potentialités de digestion des protéines du lait semblent donc beaucoup moins limitantes que celles de digestion de l'énergie (lipides principalement).

Chez les agneaux Ile-de-France, l'évolution de l'utilisation digestive des aliments d'allaitement SC $2 \mathrm{M}$ entre la deuxième et la quatrième semaine d'âge est du même ordre que chez les agneaux limousins, mais la dispersion des résultats en deuxième semaine est très élevée et le niveau des CUDa globalement inférieur aux deux âges à celui observé dans le cas du premier génotype, ce qui pourrait correspondre à une maturation plus lente du fonctionnement du tube digestif chez ces animaux.

Par ailleurs, l'augmentation de la concentration en matières azotées des laits (aliments $\mathrm{SC} 1 \mathrm{H}$ et $\mathrm{SC} 2 \mathrm{H}$ ) améliore nettement leur utilisation digestive chez les agneaux les plus jeunes ( 1 ou 2 semaines), quelque soit leur génotype. Ce phénomène, conforme à la majorité des observations (Walker \& Cook, 1967 ; Theriez, PatureauMirand \& Molenat, 1977) est principalement lié en première semaine à l'excellente valeur biologique des lactoprotéines ajoutées au lait et à l'accroissement important du rapport entre l'azote ingéré et l'azote endogène fécal, pratiquement inchangé ; mais, en deuxième semaine, l'excrétion d'azote fécal est considérablement réduite et prend une valeur $\left(0,14 \mathrm{~g} \mathrm{~N} / 100 \mathrm{~g}\right.$ MS ingérée) obtenue seulement en $3^{\mathrm{e}}$ semaine dans le cas 


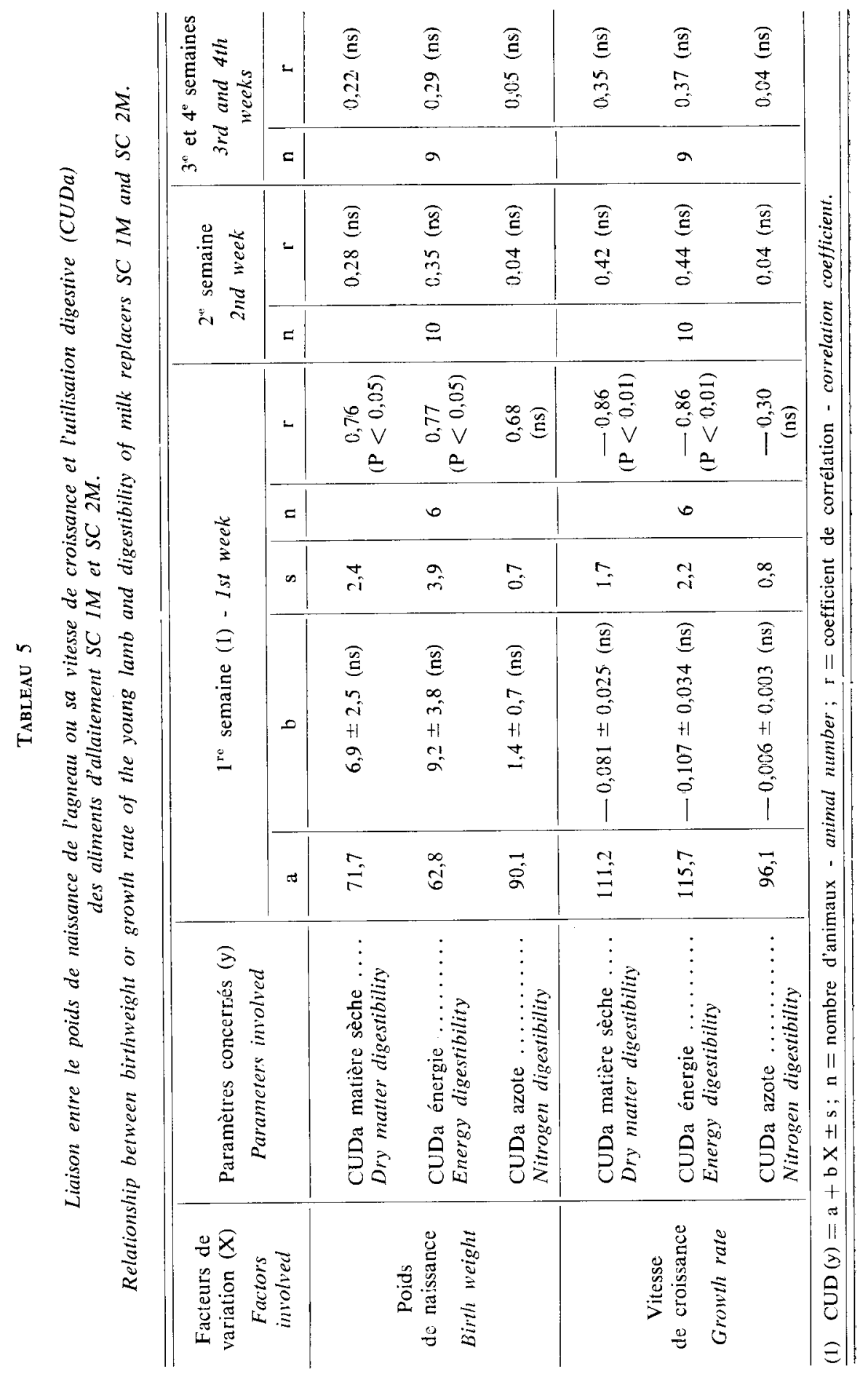


des régimes moins riches en azote (SCM). Le phénomène ne peut donc qu'imparfaitement se rattacher à des mécanismes connus : l'utilisation de régimes riches en matières azotées accroît la sécrétion de trypsine chez le jeune porc (CORRING \& SAUCIER, 1972) mais on manque d'information sur les effets de ce paramètre sur les mécanismes de digestion des lipides ou même des protéines chez le Préruminant. Dans le cas de ce type de laits enrichis en matières azotées, la digestibilité des principes alimentaires (MS, E, N) n'est significativement reliée au poids de naissance des animaux ou à leur vitesse de croissance, à aucun moment de leur vie.

Enfin, le remplacement d'une partie du suif de l'aliment d'allaitement par de la tricaproïne permet d'obtenir une digestibilité très élevée dès l'âge d'une semaine, et du même ordre que celle observée, chez des agneaux plus âgés, dans le cas où l'huile cle coprah est l'unique source de matières grasses du lait (WALKER \& STOKEs, 1970 ; THERIEz et al., 1973). Les coefficients d'utilisation digestive sont du même ordre que ceux observés avec du lait de brebis (Hodge, 1965 ; JAGUSCH \& MITChELL, 1971) ou du lait de vache (Walker \& Kirk, 1975 chez l'agneau; Toullec \& Mathieu, 1969 chez le veau).

L'amélioration du CUDa de l'énergie s'explique en grande partie par l'excellente digestibilité des acides gras à chaîne moyenne : 100,98 et 94 p. 100 respectivement pour les acides gras dont la chaîne comporte moins de 12 atomes de carbone, pour l'acide laurique (12 atomes de carbone) ou pour l'acide myristique (14 atomes de carbone) (Walker \& Stokes, 1970 ; Gibney \& Walker, 1977). Cependant, l'amplitude des effets observés a été du même ordre, indépendamment des proportions d'acide caproïque finalement présentes dans les ingesta, 4,5 p. 100 de MS (aliments SCT $1 \mathrm{M}$ et SCT 1H) ou 11,4 p. 100 de MS (aliments SCT 2M ou SCT 2H). L'accroissement des proportions de protéines, relativement à l'énergie ingérée après qu'une partie de la tricaproïne ait surnagé dans le premier cas (lait SCT 1M) est un élément d'explication possible de l'absence d'effet du taux d'acide caproïque dans le lait ingéré.

Par ailleurs, l'amélioration de la digestibilité apparente de l'azote des laits enrichis en acides gras à chaîne moyenne, déjà observée antérieurement aussi bien chez l'agneau (Walker \& Stokes, 1970; Theriez et al., 1973) que chez le veau (Roy et al., 1973 ; Aurousseau, Vermorel \& Bouvier, 1983) montre un effet des AGCM sur l'efficacité du fonctionnement du tube digestif. L'excrétion totale d'azote fécal est réduite avec les régimes «SCT », dès la première semaine à $0,12 \mathrm{~g}$ pour $100 \mathrm{~g}$ de MS ingérée, valeur sensiblement inférieure à celle indiquée par BLACK, PEARCE \& Tribe (1973) pour l'excrétion d'azote métabolique fécal $(0,20 \mathrm{~g}$ pour $100 \mathrm{~g}$ de MS ingérée) ce qui souligne l'excellent fonctionnement du tube digestif des animaux correspondants. La libération et l'absorption de l'acide caproïque dans la caillette (Edwards-WebB \& Thompson, 1978) pourrait donc appauvrir en globules gras le caillot formé par la coagulation des caséines du lait et faciliter ainsi une dégradation plus poussée des protéines dans cet organe et favoriser finalement leur utilisation digestive (Ternouth et al., 1975). Comme dans le cas précédent (laits enrichis en matières azotées), aucune liaison entre le poids de naissance des animaux ou leur vitesse de croissance et la digestibilité des principes alimentaires n'est observée.

La présence d'acide caproïque dans les aliments exerce des effets d'amplitude plus importante chez l'agneau que ceux rapportés précédemment chez le veau (Aurousseau, Vermorel \& Bouvier, 1983). Mais, dans ce cas, la digestibilité des aliments était plus faible, probablement en raison d'une détérioration partielle des acides gras insaturés 
apportés par le suif au cours des différentes étapes de la préparation ct de la conservation des laits, plus nombreuses et plus longues dans le cas de l'expérimentation sur veaux.

\section{Conclusion}

La présence de tricaproïne (en l'état ou sous forme intérestérifiée à des triglycérides d'acides gras longs) dans les aliments d'allaitement offerts au jeune agneau permet d'obtenir une digestibilité maximale de la matière sèche, de l'énergie et de l'azote apportés, dès la première semaine de vie des animaux, alors que le maximum n'est atteint qu'à l'âge de 3 à 4 semaines dans le cas des aliments d'allaitement conventionnels. L'utilisation de ce type de matières grasses permet, en comparaison de l'effet obtenu en augmentant la concentration en matières azotées des laits, une amélioration de leur digestibilité plus importante et plus précoce.

Ce triglycéride d'acide gras à chaîne moyenne permet, ainsi, de résoudre les problèmes soulevés par les limites digestives du jeune préruminant. Ses effets sur l'utilisation pour la croissance de l'énergie et de l'azote ingérés seront présentées dans une prochaine communication.

Accepté pour publication en juillet 1983.

\section{Summary \\ Combined effects of caproic acid and protein level in the milk replacers offered to preruminant lambs}

\section{1 - Digestibility of the main components of the diet}

The digestibility of milk replacer as affected by type of lipid used (tallow-coconut oil, "SC », or tallow-coconut oil-tricaproin, «SCT»), protein level (medium, « M », i.e. 24 p. 100 of dry matter, «DM», or high, « $\mathrm{H} »$, i.e. 31 p. 100 of $\mathrm{DM}$ ) or amount of tricaproin 14.5 or 11.4 p. 100 of DM) were compared on 56 preruminant male lambs in 3 successive trials (fig. 1). Digestibility was measured at 2 and 5 weeks (trial 1, 16 lambs from the limousine breed), at 1,2 and 3 weeks (trial 2, 24 male lambs from the limousine breed) or at 2 and 4 weeks (trial 3, 16 male lambs from the Ile-de-France breed).

In the case of $" \mathrm{SC} \gg$ milk replacers, the digestibility of $\mathrm{DM}, \mathrm{E}$ (energy), and $\mathrm{N}$ (nitrogen) increased linearly $(\mathrm{P}<0.005)$ from 1 week $(95.5,94.6$ and 95.1 p. 100 respectively) up to 3 weeks $(98.0,97.6$ and 97.0 respectively) .For the same diets, the digestibility of the three components increased with the protein level $(P<0.05)$ in the younger animals (1 and 2 weeks) from $95.3,94.2$ and $95.0 \mathrm{p} .100$ to $96.8,97.1$ and $96.3 \mathrm{p} .100$ respectively for the « $\mathrm{SCM} »$ or « $\mathrm{SCH} »$ milk replacers.

In the case of the "SCT " milk replacers, the digestibility was not related to the age of the animals or the protein level of the diet; compared to the values observed in the case of $« \mathrm{SC} \gg$ milk replacers, digestibility was increased $(P<0.05)$ in the younger animals ( 1 and 2 weeks of age) from $96.1,95.6$ and 95.5 p. 100 up to $98.4,98.3$ and 97.0 p. 100 respectively DM, E and $\mathrm{N}$ (tabl. 3, fig. 2, tabl. 4).

In the case of «SCM » milk replacers and in 1 week old lambs, the digestibility of DM, $E$ and $N$ were positively correlated with birthweight $(r=0.70)$ and that of $D M$ and $E$ 
were negatively correlated with growth rate of the animals $(r=-0.86)$. In all other cases (other ages as well as other diets) no relation was observed between birthweight or growth rate of the lambs and digestibility of the milk replacers (tabl. 5).

\section{Références bibliographiques}

Aurousseau B., Vermorel M., Bouvier J.C., 1983. Influence du remplacement d'une partie du suif d'un aliment d'allaitement par de la tricaproïne ou de l'huile de coprah sur l'utilisation de l'énergie ou de l'azote ingérés par le jeune veau. Effets du niveau d'alimentation. Reprod. Nutr. Dev., 23, 587-598.

Black J.L., Pearce G.R., Tribe D.E., 1973. Protein requirements of growing lambs. Br. J. Nutr., 30, 45-60.

Corring T., Saucier R., 1972. Sécrétion pancréatique sur porc fistulé. Adaptation à la teneur en protéines du régime. Ann. Biol. anim. Biochim. Biophys., 12, 233-241.

EDWARDS-WEBB J.D., ThOMPSON S.Y., 1977. Studies on lipid digestion in the preruminant calf. 2 - A comparison of the products of lipolysis of milk fat by salivary and pancreatic lipases in vitro. Br. J. Nutr., 37, 431-440.

EDWARDS-WEBb J.D., Thomson S.Y., 1978. Studies on lipid digestion in the preruminant calf. 3 - The action of salivary lipase on milk fat in the abomasum. Br. J. Nutr., 40, $125-131$.

Entressangles B., Paserol L., Savary P., Sarda L., Desnuelle P., 1961. Influence de la nature des chaînes grasses sur la vitesse de leur hydrolyse par la lipase pancréatique. Bull. Soc. Chim. biol., 43, 581-591.

Gibney M.J., Walker D.M., 1977. Milk replacers for preruminant lambs : protein and fat interactions. A ust. J. agric. Res., 28, 703-712.

Guilloteau P., Corring T., Garnot P., Martin P., Toullec R., Durand G., 1983 a. Effects of age or weaning on enzyme activities of abomasum and pancreas in the lamb. J. Dairy Sci. (sous presse).

Guilloteau P., Corring T., Toullec R., Robelin J., 1983 b. Effects of age on enzyme activities of abomasum and pancreas in the preruminant calf. Proc. 4th Intern. Symp. on Protein and Metabolism nutrition, Clermont-Ferrand (sous presse).

HODgE R.W., 1965. The apparent digestibility of ewes milk by young lambs. Aust. J. exp. Agric. anim. Husbe, 5, 369-371.

Houssin Y., Davicco M.J., 1979. Influence of birth weight on the digestibilty of a milk replacer in newborn-lambs. Ann. Rech. vet., 10, 419-421.

JaGusCh K.T., Mitchell R.M., 1971. Utilization of metabolizable energy of ewes milk by the lambs. N.Z. agric. Res., 14, 434-441.

LEat W.M.F., Harrison F.A., 1975. Digestion, absorption and transport of lipids in the sheep. In : Mc Donald I.W., WARNER A.C.I., Digestion and metabolism in the ruminant. The University of New-England Publishing unit, Armidale, 481-495.

Norton B.W., Walker D.M., 1971. Nitrogen balance studies with the milk-fed lamb. 7 - Effect of age of the lamb. Br. J. Nutr., 26, 1-6.

Paruelle J.L., Toullec R., Frantzen J.F., Mathieu C.M., 1972. Utilisation des protéines par le veau préruminant à l'engrais. I - Utilisation digestive des protéines de soja et des levures d'alcanes incorporées dans les aliments d'allaitement. Ann. Zootech., 21, 319-331.

Patureau-Mirand P., Theriez M., 1977. Amino acid requirement of preruminant lambs (abstr.). Ann. Zootech., 26, 287.

Roy J.H.B., Stobo I.J.F., Gaston H.J., Shotton S.M., Ganderton P.. 1973. The nutrition of the veal calf. 5 - Comparison of two margarine fats. Anim. Prodl., 17, 97-107.

Snedecor G.W., Cochran W.G., 1971. Méthodes statistiques. Trad. H. Boelle et E. CamMaJi, Association de coordination technique agricole, Paris, Ed., 524-559. 
Ternouth J.H., Roy J.H.B., 1973. The effect of diet and feeding technique on digestive function in the calf. Ann. Rech, vet., 4, 19-30.

Ternouth J.H., Roy J.H.B., Thompson S.Y., Toothill J., Gillies C.M., Edwards-Werb J.D., 1975. Concurrent studies of the flow digesta in the duodenum and exocrine pancreatic secretion of calves. 3 - Further studies on the addition of fat to skim milk and the use of non milk protein in milk substitute diets. Br. J. Nutr., 31, 181-196.

Ternouth J.H., Siddons R.C., Toothill J., 1971. Pancreatic secretion in the milk-fed calf. Proc. Nut. Soc., 30, 89 A.

Theriez M., Molenat G., Daniel M., Aurousseau B., 1973. L'allaitement artificiel des agneaux. III - Influence de la nature des matières grasses incorporées dans l'aliment d'allaitement. Ann. Zootech., 22, 185-197.

Theriez M., Patureau-Mirand P., Molenat G., 1977. Allaitement artificiel de l'agneau. VI - Comparaison de différentes teneurs en matières azotées du lait de remplacement. Ann. Zootech., 26, 297-313.

Theriez M., Protais M., Molenat G., 1974. L'allaitement artificiel des agneaux. IV Comparaison de différentes sources de matières azotées en remplacement de la poudre de lait. Ann. Zootech., 23, 325-341.

Tissier M., Bechet G., Molenat G., 1975. Appareils de collecte totale des fèces pour agneaux à l'allaitement ou à l'engrais ou pour brebis. Ann. Zootech., 24, 595-602.

Toullec R., Frantzen J.F., Mathieu C.M., 1974. Influence de la coagulation des protéines du lait sur l'utilisation digestive d'un lait de remplacement par le veau préruminant. Ann. Zootech., 23, 359-364.

Toullec R., Mathieu C.M., 1969. Utilisation digestive des matières grasses et de leur principaux acides gras par le veau préruminant à l'engrais. Influence sur la composition corporelle. Ann. Biol. anim. Biochim. Biophys., 9, 139-160.

WalKer D.M., COOK L.Y., 1967. Nitrogen balance studies with the milk fed lamb. 4 - Effect of different nitrogen and sulphur intakes on live weight gain and wool and on nitrogen and sulphur balances. Br. J. Nutr., 21, 237-256.

WALKer D.M., KIRK R.D., 1975. Methionine supplementation of milk proteins for preruminant lambs. I - Effect of protein concentration and source of carbohydrate on nitrogen balance. Aust. J. agric. Res., 26, 673-679.

Walker D.M., STOKES G.B., 1970. The nutritive value of fat in the diet of the milk fed lamb. 1 - The apparent and corrected digestibilities of different fats and their constituent fatty acids. Br. J. Nutr., 24, 425-433. 